\title{
Heartbeat: improving risk prediction and diagnosis of aortic dissection
}

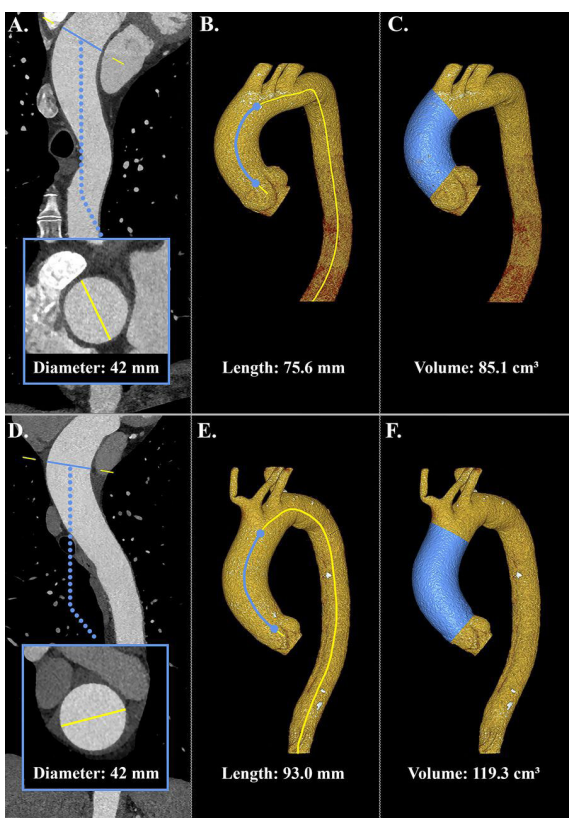

Figure 1 Methodology of the three different aortic measurements in a patient with TAA and a patient with pre-ATAAD. $(\mathrm{A}-\mathrm{C})$ demonstrate aortic measurements in a patient with TAA. panel a depicts a stretched view of the curved planar CT reconstruction with diameter measurement perpendicular to the centreline. the two right panels display a 3D CT model with measurements of ascending aortic length (B) and volume (C). (D)-(F) demonstrate similar measurements in a patient with pre-ATAAD. despite equal diameters, the patient with preATAAD exhibits a notably longer and larger ascending aorta. ATAAD, acute type $A$ aortic dissection; TAA, thoracic aortic aneurysm; 3D, three-dimensional.

In patients at risk of ascending aortic dissection, timing of surgery typically is based on serial imaging measurements of aortic diameter. Clearly, the risk of dissection in an individual patient is higher at larger aortic diameters. Even so, from a population point-of-view, most acute dissections occur in patients with a diameter below the recommended threshold for prophylactic root replacement. In this issue of Heart, Heuts and colleagues ${ }^{1}$ evaluated the hypothesis that measures of

Correspondence to Professor Catherine M Otto, Division of Cardiology, University of Washington, Seattle, Washington, USA; cmotto@uw.edu aortic length and volume would be better predictors of the risk of dissection than diameter alone. (figure 1) In an observation cohort study of 477 consecutive patients with a Type A aortic dissection, $96 \%$ did not meet the surgical diameter threshold of $55 \mathrm{~mm}$ before dissection onset. Compared with a cohort 75 patients with an aortic aneurysm who did not suffer a dissection, the positive predictive values of aortic measurements for prediction of dissection were $20 \%$ for maximal aortic diameter, $55 \%$ for aortic volume and $70 \%$ for aortic length.

In the accompanying editorial, Akin and Nienaber $^{2}$ emphasise the need to consider other risk factors for aortic dissection, not just aortic size. Hypertension is the most common risk factor across the age spectrum. Additional risk factors in older patients include atherosclerosis, prior cardiac surgery or a known aortic aneurysm whereas in younger patients the predominant risk factors are genetic conditions. They also note that the current study comparing patients with an aortic aneurysm who did or did not suffer an aortic dissection builds on the author's previous studies describing the normal changes in aortic length with ageing and showing that excessive elongation of the ascending aorta is a predictor of aortic dissection. ${ }^{34}$ Akin and Nienabaer conclude that "Dimensional anatomical parameters such as diameter, length and volume are certainly factors to be considered in the predictive analysis of aortic dissection. However, these parameters often represent the result of long-lasting influences of hypertension or connective tissue disorders, and a prediction tool based on anatomy only would leave many aspects unconsidered (figure 2).'

Continuing on the theme of aortic disease, a state-of-the-art review article by Salmasi and colleagues ${ }^{5}$ highlights the rapidity of the disease course and high rate of adverse outcomes in patients with an acute aortic dissection, focusing on factors that might result in a delayed or missed diagnosis. The authors recommend prompt assessment of the risk of aortic dissection and then proceeding rapidly to definitive diagnosis with cross-sectional (usually computed tomographic) imaging. (figure 3) Another useful resource for improving diagnosis of acute aortic dissection at your own institution is the article by Carroll and colleagues ${ }^{6}$ on imaging for acute aortic syndromes.

The Education in Heart article in this issue, written by my genetic colleagues and myself, provides a "primer" on cardiogenetics for clinical cardiologists. This introductory article outlines the approach to diagnosis of inherited cardiac

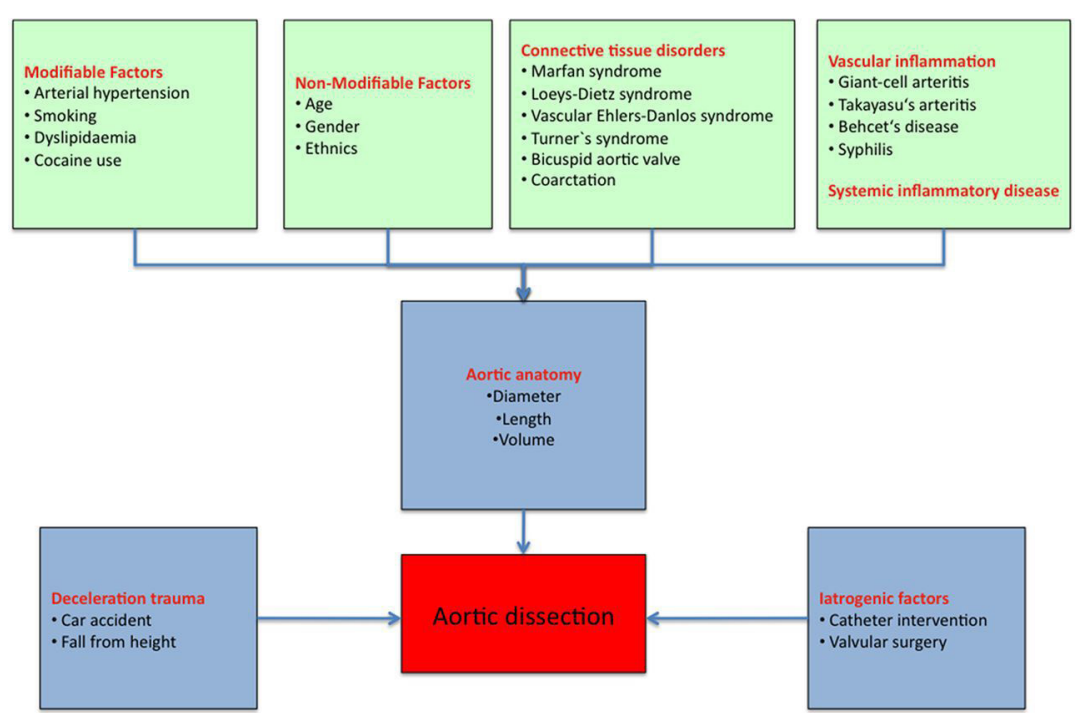

Figure 2 Factors influencing the aortic dimensions and risk of aortic dissection in general. 


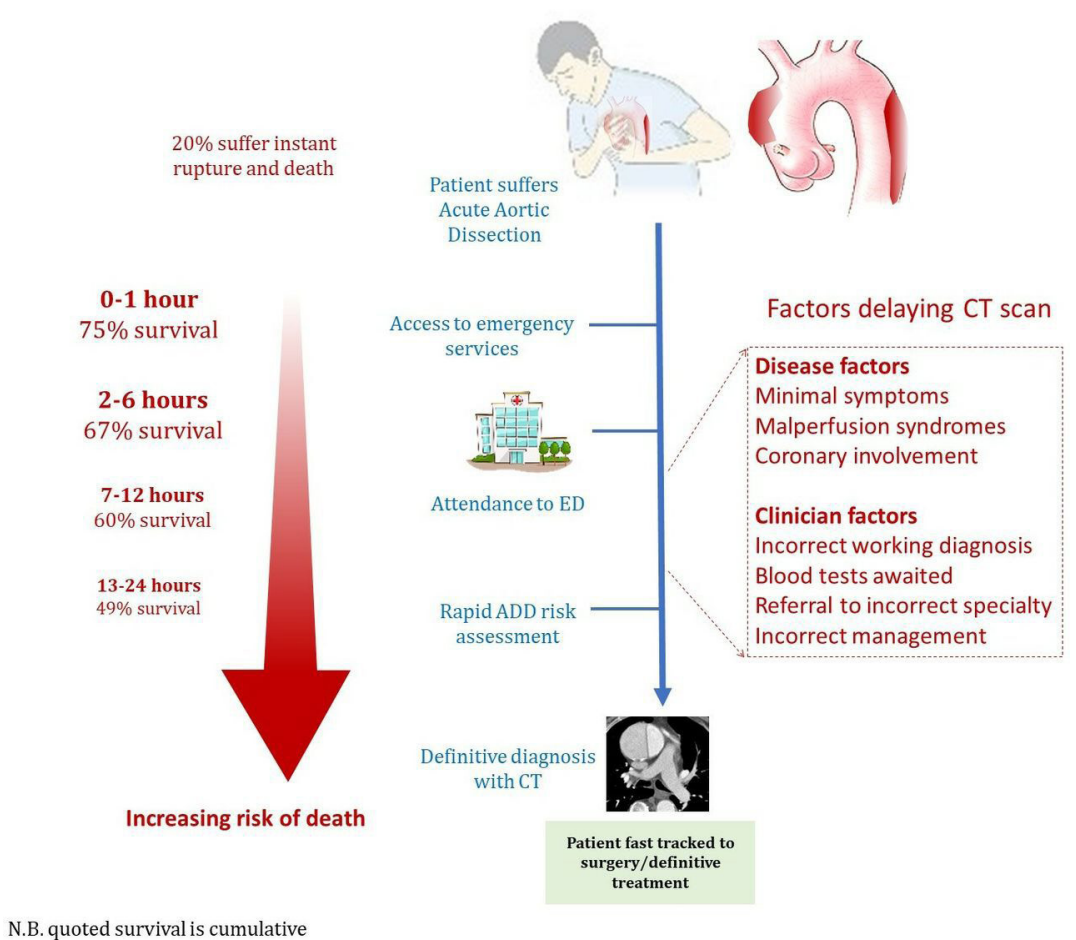

Figure 3 Risk factors for misdiagnosis in aortic dissection. AD, aortic dissection; ED, emergency department. conditions based on the clinical presentation, family history and directed genetic testing. Detailed tables summarise the relationship between genotype and clinical disease and how specific genetic data affects patient management for aortic disease, cardiomyopathies and cardiac arrhythmias. Particularly for inherited aortic conditions, earlier diagnosis and genetic testing will allow prevention of acute aortic dissection in many individuals and families. Articles in future issues of Heart will discuss specific inhertied cardiac conditions in more detail.

Management of patients with heart failure with reduced ejection fraction and secondary mitral regurgitation (MR) remains controversial. One of the challenges is accurate diagnosis of MR severity with considerable measurement variability in the recommended Doppler quantitative measures. Kamoen and colleagues ${ }^{8}$ demonstrate the validity of a simpler and reproducible method for quantitation of

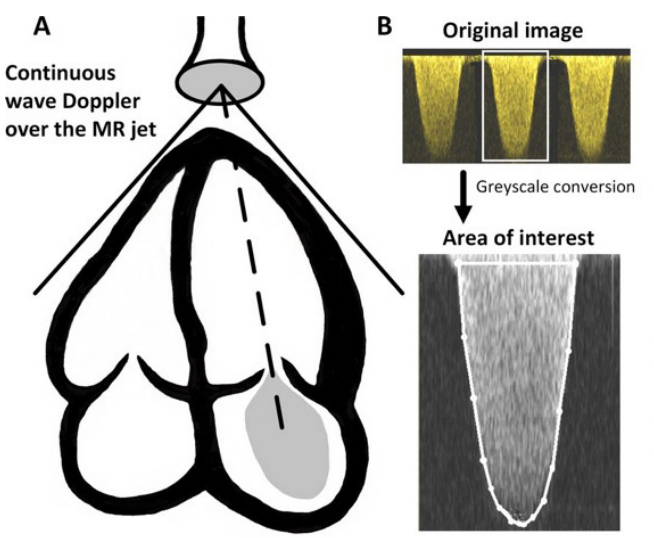

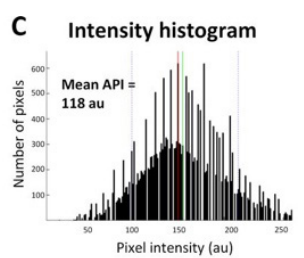

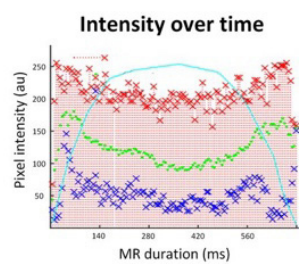

Figure 4 Acquisition and calculation of the API. panel A: for the API acquisition, a continuous wave Doppler is performed over the mitral valve, well aligned with the mitral regurgitation jet. panel B: the uncompressed image is stored and converted to greyscale. The operator manually traces the area of interest, that is, the continuous wave Doppler envelope. panel C: using specific software, the API value is calculated and pixel intensities over time are displayed. API, average pixel intensity.
MR severity based on the average pixel intensity (API) of the continuous-wave Doppler signal (figure 4). In an editorial, Kusunose" comments "The API has advantages including quantitative, single measurement, and avoid geometric assumptions. Moreover, the API method is a simple digital format and can be suitable in the modern era of artificial intelligence of medical imaging."

In patients undergoing transcatheter mitral valve repair (TMVR), a subset of patients has a normal left atrial pressure (LAP) despite severe MR. Sims and colleagues ${ }^{10}$ found a normal LAP in $15 \%$ of 204 patients undergoing TMVR. Clinical and hemodynamics parameters were similar between groups with the exception that normal LAP was associated chronic lung disease with on multivariable analysis (OR 4.79 (1.83-12.36), $\mathrm{p}=0.001)$. In the editorial commenting on this study, Minder and Whisenant ${ }^{11}$ hypothesise that "Data presented by Sims et al, suggest that left atrial compliance, in addition to mitral regurgitant volume, may play a critical role in predicting which patients benefit from mitral intervention." They then propose several questions about management of patients with severe MR that deserve attention in future clinical trials.

Funding The authors have not declared a specific grant for this research from any funding agency in the public, commercial or not-for-profit sectors.

Competing interests None declared.

Patient and public involvement Patients and/ or the public were not involved in the design, or conduct, or reporting, or dissemination plans of this research.

Patient consent for publication Not required.

Provenance and peer review Commissioned; internally peer reviewed.

(C) Author(s) (or their employer(s)) 2020. No commercial re-use. See rights and permissions. Published by BMJ.

\section{(D) Check for updates}

To cite Otto CM. Heart 2020;106:867-869.

Heart 2020;106:867-869.

doi:10.1136/heartjnl-2020-317283

ORCID iD

Catherine M Otto http://orcid.org/0000-0002-05279392

\section{REFERENCES}

1 Heuts S, Adriaans BP, Rylski B, et al. Evaluating the diagnostic accuracy of maximal aortic diameter, length and volume for prediction of aortic dissection. Heart 2020;106:892-7.

2 Akin I, Nienaber CA. Prediction of aortic dissection. Heart 2020;106:870-1. 
3 Adriaans BP, Heuts S, Gerretsen S, et al. Aortic elongation Part I: the normal aortic ageing process. Heart 2018;104:1772-7.

4 Heuts S, Adriaans BP, Gerretsen S, et al. Aortic elongation Part II: the risk of acute type A aortic dissection. Heart 2018;104:1778-82.

5 Salmasi MY, Al-Saadi N, Hartley P, et al. The risk of misdiagnosis in acute thoracic aortic dissection: a review of current guidelines. Heart 2020;106:885-91.
6 Carroll BJ, Schermerhorn ML, Manning WJ. Imaging for acute aortic syndromes. Heart 2020;106:182-9.

7 Otto CM, Savla JJ, Hisama FM. Cardiogenetics: a primer for the clinical cardiologist. Heart 2020;106:938-47.

8 Kamoen V, De Buyzere M, El Haddad M, et al. Average pixel intensity method for prediction of outcome in secondary mitral regurgitation. Heart 2020;106:904-9.
9 Kusunose K. What is a better measure of regurgitant severity in secondary mitral regurgitation by echocardiography? Heart 2020;106:874-5.

10 Sims JR, Reeder GS, Guerrero M, et al. Characteristics and outcomes of patients with normal left atrial pressure undergoing transcatheter mitral valve repair. Heart 2020;106:898-903.

11 Minder CM, Whisenant BK. Severe mitral regurgitation: does one size fit all? Heart 2020;106:872-3. 